\section{Subclinical thyroid disorders}

No large randomised controlled trials in subclinical hyperthyroidism nor subclinical hypothyroidism have used robust clinical endpoints, particularly cardiovascular morbidity and mortality. Despite this absence of evidence, David Cooper and Bernadette Biondi (March 24, p 1142) ${ }^{1}$ make rather strong recommendations favouring treatment in both situations, particularly for patients with concentrations of thyroid-stimulating hormone below $0.1 \mathrm{mU} / \mathrm{L}$ or above $5.0 \mathrm{mU} / \mathrm{L}$.

As mentioned in their Introduction, subclinical thyroid diseases are defined on a biological basis, might but might not always constitute an early stage of "overt" thyroid diseases, and are most often completely asymptomatic. Both disorders represent more of a risk situation than a disease and the therapeutic stake is not to improve the clinical status of patients but to prevent risk (mainly of atrial fibrillation in subclinical hyperthyroidism and coronary events in subclinical hypothyroidism). Consequently, it seems crucial to ensure that treatment will not have a deleterious effect that could outweigh any potential benefit.

Clinicians have to keep in mind that treatment of subclinical hyperthyroidism, particularly with radioiodine, carries a great risk of inducing definite hypothyroidism. Several epidemiological studies ${ }^{2-4}$ have shown that less than $60 \%$ of patients treated with thyroxine reach euthyroid status, the others having either persistent hypothyroidism (generally subclinical) or hyperthyroidism (owing to overtreatment). It seems rather paradoxical in a Seminar on both disorders not to mention that treatment of one situation has a great chance of leading to the other one.

As mentioned in the linked Editorial, ${ }^{5}$ randomised controlled trials are urgently needed. It would have been interesting to discuss why doctors, academic researchers, and public health authorities invest so little in doing them.

BG and SV contributed equally to the writing. BG has been speaker at symposia organised by Merck Serono. SV declares that he has no conflicts of interest.

\section{*Bernard Goichot, Stéphane Vinzio} bernard.goichot@chru-strasbourg.fr

Service de Médecine Interne et Nutrition, Hôpital de Hautepierre, Hopitaux Universitaires de Strasbourg, 67098 Strasbourg, France (BG); and Groupe Hospitalier Mutualiste de Grenoble, Grenoble, France (SV)

1 Cooper DS, Biondi B. Subclinical thyroid disease. Lancet 2012; 379: 1142-54.

2 Parle JV, Franklyn JA, Cross KW, Jones SR, Sheppard MC. Thyroxine prescription in the community: serum thyroid stimulating hormone level assays as an indicator of undertreatment or overtreatment. BrJ Gen Pract 1993; 43: 107-09.

3 Canaris GJ, Manowitz NR, Mayor G, Ridgway EC. The Colorado thyroid disease prevalence study. Arch Intern Med 2000; 160: 526-34.

4 Turner MR, Camacho X, Fischer HD, et al. Levothyroxine dose and risk of fractures in older adults: a nested case-control study. BMJ 2011; 342: d2238.

5 The Lancet. Thyroid disease-more research needed. Lancet 2012; 379: 1076.

Subclinical hypothyroidism is prevalent in older age, but the clinical implications are uncertain. David Cooper and Bernadette Biondi ${ }^{1}$ recommend treatment of mild subclinical hypothyroidism, but make an exception for those older than 75 years. First they argue that there is insufficient evidence to support an association with symptoms and that replacement does not improve cognition or quality of life. Second, they refer to an association between subclinical hypothyroidism and better survival in the oldest old. We interpret the evidence differently.

Studies on the association between subclinical hypothyroidism and various symptoms and signs have shown equivocal results. The largest metaanalysis $^{2}$ reports an association between subclinical hypothyroidism and cardiovascular disease, but no evidence for age specificity. Randomised controlled trials have been heterogeneous, underpowered, and have included few very old participants. The Cochrane review ${ }^{3}$ therefore recommends more trials, especially in this group.

In one population-based study of individuals aged 85 years or older, subclinical hypothyroidism was associated with better survival. ${ }^{4}$ However, observational results should be interpreted with caution. Other risk factors, such as high blood pressure and high cholesterol, also associate with better survival in the highest age group, but randomised controlled trials have still shown benefits from treatment of these risk factors.

We believe that the available evidence does not support an agespecific treatment recommendation. Cooper and Biondi describe an "urgent need" for randomised clinical trials. A nationwide initiative launched by our institute will start such a trial. In participants aged 80 years and older, we will study the effect of treatment on several endpoints ranging from cardiovascular disease and mortality to cognitive and physical function and quality of life.

We declare that we have no conflicts of interest.

Simon P Mooijaart, on behalf of the IEMO 80-plus Thyroid Trial

Collaboration

mooijaart@iemo.nl

Institute for Evidence-based Medicine in Old Age (IEMO), 2333 AA Leiden, Netherlands

1 Cooper DS, Biondi B. Subclinical thyroid disease. Lancet 2012; 379: 1142-54.

2 Rodondi N, den Elzen WPJ, Bauer DC, et al. Subclinical hypothyroidism and the risk of coronary heart disease and mortality. JAMA 2010; 304: 1365-74

3 Villar HC, Saconato H, Valente O, Atallah AN. Thyroid hormone replacement for subclinical hypothyroidism. Cochrane Database Syst Rev 2007; 3: CD003419.

4 Gussekloo J, van Exel E, de (raen AJ Meinders AE, Frölich M, Westendorp RG. Thyroid status, disability and cognitive function, and survival in old age. JAMA 2004; 292: 2591-99.

David Cooper and Bernadette Biondi ${ }^{1}$ comment that guidance on treatment of subclinical hypothyroidism has been "well-meaning but necessarily vague". However their clinical recommendations are based on epidemiological associations, rather than
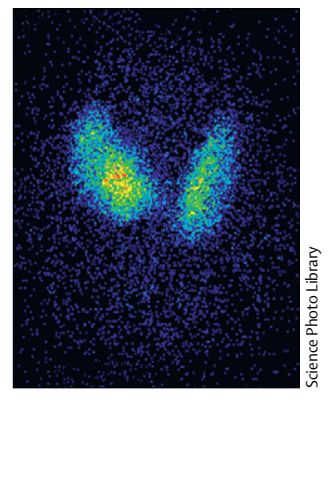


\section{Correspondence}

evidence from randomised controlled trials.

Two specific recommendations give particular concern: (1) "Treatment with levothyroxine is recommended in...patients with subclinical hypothyroidism with serum TSH concentrations of $10 \mathrm{mU} / \mathrm{L}$ or higher" and (2) "Treatment of mild subclinical hypothyroidism is not recommended in elderly...patients."

Cohort studies show increased cardiovascular mortality associated with concentrations of thyroidstimulating hormone (TSH) of 10.0-19.9 $\mathrm{mU} / \mathrm{L}$ (hazard ratio adjusted for age and sex 1.58, 95\% Cl 1.10-2.27; compared with TSH 4.5-6.9 mU/L). ${ }^{2}$ However, subclinical hypothyroidism in those older than 80 years is associated with improved health and survival compared with the euthyroid state. ${ }^{3}$ These associations are not sufficient to prove cause, and cannot fully inform decisions for clinical practice.

The Cochrane review of thyroxine replacement for subclinical hypothyroidism ${ }^{4}$ (12 trials, 350 patients) found insufficient evidence to allow definitive advice for prescribing (or avoiding) treatment, including in subgroups of those with TSH concentrations above $10 \mathrm{mU} / \mathrm{L}$, or in very elderly people. In the absence of high-quality evidence from randomised controlled trials, there is clinical equipoise and we strongly endorse Cooper and Biondi's conclusion that further trials are required.

Funded by the EU, we are soon to start the Thyroid Hormone Replacement for Untreated older adults with Subclinical hypothyroidism Trial

(TRUST)-a multicentre $(\mathrm{n}=3000)$, randomised, placebo-controlled trial of thyroxine in subclinical hypothyroidism with cardiovascular and quality-of-life outcomes.

Definitive recommendations should await the results of randomised controlled trials such as TRUST. Premature guidance risks causing harm, and might negatively affect trials that could answer these clinical questions by limiting the number of patients available for recruitment.

All authors are grant holders on TRUST.

*Terence J Quinn, Jacobijn Gussekloo, Patricia Kearney, Nicolas Rodondi, David J Stott

\section{terry.quinn@glasgow.ac.uk}

University of Glasgow, Glasgow Royal Infirmary, Glasgow G4 OSF, UK (TJQ, DJS); Leiden University Leiden, Netherlands (JG); University College Cork, Cork, Ireland (PK); and University of Bern, Bern, Switzerland (NR)

1 Cooper DS, Biondi B. Subclincal thyroid disease. Lancet 2012; 379: 1142-54.

2 Rodondi N, den Elzen WP, Bauer DC, et al. Subclinical hypothyroidism and the risk of coronary heart disease and mortality. JAMA 2010; 304: 1365-74

3 Gussekloo J, van Exel E, de Craen AJ, Meinders AE, Frölich M, Westendorp RG. Thyroid status, disability and cognitive function, and survival in old age. JAMA 2004; 292: 2591-99.

4 Villar HC, Saconato H, Valente O, Atallah AN. Thyroid hormone replacement for subclinical hypothyroidism. Cochrane Database Syst Rev 2007; 3: CD003419.

\section{Authors' reply}

Bernard Goichot and Stéphane Vinzio state that we make "rather strong recommendations" favouring treatment of subclinical thyroid dysfunction. Our recommendations to treat were for patients with the most extreme degrees of subclinical hyperthyroidism and hypothyroidism (serum thyroid-stimulating hormone [TSH] $<0.1 \mathrm{mU} / \mathrm{L}$ and $>10 \mathrm{mU} / \mathrm{L}$, respectively) and to "consider" individualised treatment for milder degrees of thyroid impairment, taking the patient's age and associated risk factors into account. Obviously, the treatment should not be worse than the disease. Goichot and Vinzio correctly point out that both mild subclinical hyperthyroidism and subclinical hypothyroidism represent risk factors for future comorbidity rather than diseases, although some patients do have mild symptoms, and subclinical hypothyroidism in pregnancy is clearly associated with many adverse outcomes.

We recommend treatment of subclinical hyperthyroidism when serum TSH concentrations are below $0.1 \mathrm{mU} / \mathrm{L}$, in accord with others, ${ }^{1}$ and on the basis of the risks, especially in older people, that we discuss in our paper. As with anything in medicine, the risks and benefits of any intervention need to be assessed and we believe that the risk/benefit ratio for treatment is favourable. There is evidence, some of it new, that subclinical hyperthyroidism, especially in the presence of undetectable serum $\mathrm{TSH}$, might cause atrial fibrillation, fractures, stroke, and increased mortality. ${ }^{2}$ The high risk associated with subclinical hyperthyroidism, particularly in older people, favours intervention, keeping in mind that iatrogenic hypothyroidism is possible, and that thyroid hormone replacement therapy might be needed.

We agree that many studies have shown that treatment of hypothyroidism with thyroid hormones is often not optimised, leading to inadvertent overtreatment or undertreatment. To decrease the frequency of permanent hypothyroidism, we do note in our Seminar that radioiodine is preferred in patients with toxic multinodular goitres, and antithyroid agents are preferred in patients with Graves' disease.

Simon Mooijaart and Terence Quinn and colleagues note planned clinical trials to assess the risks and benefits of treating subclinical hypothyroidism in patients older than 80 years. This is, of course, good news. However, until the results of these trials are available, which is likely to be many years from now, clinicians are in need of recommendations based on the best available evidence. As Mooijaart points out, serum TSH concentrations above $10 \mathrm{mU} / \mathrm{L}$ have been associated with adverse cardiovascular outcomes in many studies, including in older people, although this finding remains controversial in individuals aged 85 years and older, especially regarding the risk of coronary heart disease. However, in elderly people with subclinical hypothyroidism, 